\title{
Epi-detecting label-free multimodal imaging platform using a compact diode-pumped femtosecond solid-state laser
}

Marco Andreana

Tuan Le

Anders K. Hansen

Aart J. Verhoef

Ole B. Jensen

Peter E. Andersen

Paul Slezak

Wolfgang Drexler

Alma Fernández

Angelika Unterhuber 


\title{
Epi-detecting label-free multimodal imaging platform using a compact diode-pumped femtosecond solid-state laser
}

\author{
Marco Andreana, ${ }^{a}$ Tuan Le, ${ }^{b}$ Anders K. Hansen, ${ }^{c, d}$ Aart J. Verhoef, ${ }^{a, e}$ Ole B. Jensen, ${ }^{c}$ Peter E. Andersen, ${ }^{c}$ \\ Paul Slezak, ${ }^{f}$ Wolfgang Drexler, ${ }^{a}$ Alma Fernández, ${ }^{a, e}$ and Angelika Unterhuber, \\ aMedical University of Vienna, Center for Medical Physics and Biomedical Engineering, Vienna, Austria \\ ${ }^{b}$ Femtolasers Produktions $\mathrm{GmbH}$, Vienna, Austria \\ ${ }^{c}$ Technical University of Denmark, Department of Photonics Engineering, Roskilde, Denmark \\ dNorlase ApS, Roskilde, Denmark \\ 'Vienna University of Technology, Photonics Institute, Vienna, Austria \\ fLudwig Boltzmann Institute for Experimental and Clinical Traumatology, Vienna, Austria
}

\begin{abstract}
We have developed an epi-detected multimodal nonlinear optical microscopy platform based on a compact and cost-effective laser source featuring simultaneous acquisition of signals arising from hyperspectral coherent anti-Stokes Raman scattering (CARS), two-photon fluorescence, and second harmonic generation. The laser source is based on an approach using a frequency-doubled distributed Bragg reflector-tapered diode laser to pump a femtosecond Ti:sapphire laser. The operational parameters of the laser source are set to the optimum trade-off between the spectral and temporal requirements for these three modalities, achieving sufficient spectral resolution for CARS in the lipid region. The experimental results on a biological tissue reveal that the combination of the epi-detection scheme and the use of a compact diode-pumped femtosecond solid-state laser in the nonlinear optical microscope is promising for biomedical applications in a clinical environment. $\odot$ The Authors. Published by SPIE under a Creative Commons Attribution 3.0 Unported License. Distribution or reproduction of this work in whole or in part requires full attribution of the original publication, including its DOI. [DOI: 10.1117/1.JBO.22.9.091517]
\end{abstract}

Keywords: diode lasers; solid-state lasers; nonlinear optics; scanning microscopy; Raman spectroscopy; hyperspectral imaging. Paper 160828SSRR received Dec. 2, 2016; accepted for publication Jun. 30, 2017; published online Aug. 17, 2017.

\section{Introduction}

In recent years, a variety of label-free microscopy techniques have been developed for imaging different kinds of specimens without the use of exogenous stains or fluorophores that may cause problems in studying dynamics and living systems. ${ }^{1-6}$ Following this trend, label-free imaging is gradually being merged with classical diagnostic tools in many different fields. This mainly becomes possible due to the fact that laser technology used in nonlinear optical imaging has translated into powerful well-established, robust, and turn-key systems, which are now commercially available. Several efforts have been undertaken to bring multimodal nonlinear imaging into more clinical applications. ${ }^{7-9}$ Nonetheless, the widespread transfer to a clinical environment for routine biomedical imaging is still at an early stage. To facilitate this transition, two key aspects have to be taken into consideration in the microscopy platforms design: the detection scheme and laser technology. Especially in clinical applications, it is not possible to detect nonlinear signals in the forward propagation direction due to the thickness and the high scattering of the tissues of interest. The only possible way to detect those signals is in the backward (epi) direction which still represents a challenge due to the low intensity of the epipropagation coherent Raman-based and second harmonic signals. ${ }^{10-14}$ In terms of image acquisition, speed laser technology

*Address all correspondence to: Angelika Unterhuber, E-mail: angelika unterhuber@meduniwien.ac.at plays an important role as short pixel dwell time ensures less phototoxicity but at the same time generates lower image contrast. The ideal and universal laser source for multimodal nonlinear microscopy that meets the requirements of the most common imaging techniques, including coherent anti-Stokes Raman scattering (CARS), second harmonic generation (SHG), and two-photon fluorescence (TPF), at the same time is difficult to define in general, but optimum compromises can be reached by using femtosecond lasers. They produce very high peak powers and relatively low noise which are key factors for fast high contrast image formation, i.e., for real-time imaging. ${ }^{10,15-17}$ Indeed, TPF and SHG signals increase with respect to the intensity of the excitation pulses provided the threshold for damage and saturation is not reached. ${ }^{1,3}$ Spectral focusing CARS allows obtaining an optimum balance between spectral resolution in backward-directed hyperspectral CARS and sufficient signal intensities in TPF and SHG by changing the effective Raman width to be probed through the use of chirped pulses. ${ }^{15,18,19}$ The particular chirp value chosen depends on the sample under observation and can be changed to target the required experimental needs. Since the pulses are chirped, changing the time delay between the pump and Stokes pulses changes the instantaneous frequency difference and, therefore, the Raman mode being probed. The use of chirped pulses allows to match the effective laser linewidth with the width of the Raman resonance under consideration resulting in an improved ratio between resonant and nonresonant signals. This is more advantageous when combined with an epi-detection scheme because the amount of backward-propagating nonresonant signal depends on scatterer size and its surrounding medium (their 
difference in index of refraction). ${ }^{15,18,19}$ When using chirpmatched pulses, the interaction is maintained over a long temporal window. Temporal delay with equally chirped beams can select different molecular vibrations. In this way, hyperspectral imaging can be achieved by changing the relative delay between the interacting pulses. This method has been extensively used to image lipid content due to the high Raman cross section of these molecules in the $\mathrm{C}-\mathrm{H}$ stretching vibrations. Indeed, intracellular lipid bodies and in general lipid metabolism play an important role in human health and diseases, i.e., in cancer development. ${ }^{20}$ In addition, TPF and SHG can visualize proteins and ions by fluorescent labeling or specific autofluorescence structure, such as elastin and noncentrosymmetric molecules, such as collagen to expand the observable biological structures.

In the majority of biological and medical imaging demonstrations/applications, Ti:sapphire femtosecond light sources have been used for simultaneous TPF, SHG, and CARS microscopy. Over the last decade, femtosecond fiber lasers have also shown tremendous progress in nonlinear microscopy. ${ }^{21-23}$ Much research activity has focused on the development of these robust and compact laser sources. They offer high average powers with high beam quality and have low fabrication costs with low demands on peak power, polarization, emission band, and pulse quality. However, fiber and Ti:sapphire laser approaches face limitations in the implementation of backward-directed hyperspectral CARS combined with TPF and SHG. A bandwidth of a few nanometers is required to have enough spectral resolution to uniquely identify the Raman molecular signature of interest, i.e., to distinguish lipid and protein in the C-H vibrational region. Power levels in the range of $500 \mathrm{~mW}$ and tunability of the central wavelength are required for efficient CARS signal generation.

Ti:sapphire laser systems pumped by frequency-doubled diode-pumped solid-state lasers with high output powers and excellent beam quality have been already demonstrated in a clinical setting with TPF and SHG modalities in combination with a multiplex CARS add-on. ${ }^{9}$ One drawback, however, is that such laser systems still remain expensive and bulky preventing widespread use. Therefore, compact Ti:sapphire lasers are highly desired to facilitate the transition of this technology into a field diagnostic tool to diagnose of diseases at an early stage where molecular changes on a microscopic level occur.

Since ultrafast lasers first became commercially available in the early 1990s, the basic principle of how a mode-locked laser delivers femtosecond pulses has not undergone groundbreaking changes in the sense that their size, performance, and cost to a large extent still depend on the power, size, and cost of the available pump lasers. They are based on diode-pumped frequencydoubled solid-state lasers. In fact, the level of complexity of the pump source results in demanding manufacturing efforts. Thus, the size and cost of a femtosecond Ti:sapphire laser primarily depend on the availability and technology of the pump laser. In contrast to standard solid-state laser technology, a tapered diode laser combined with a periodically poled nonlinear crystal does not require any sensitive optical resonator to achieve SHG efficiencies up to $19 \%$ with one nonlinear crystal only in singlepass configuration. ${ }^{24}$ As alignment tolerances are significantly relieved compared to a resonator concept, innovative mounting and packaging technologies can be applied, aiming at significantly reduced costs, improved compactness, and performance with respect to low amplitude noise and long-term power stability. Using this concept, $82 \mathrm{~mW}$ from a mode-locked Ti: sapphire laser pumped by a single-pass frequency-doubled distributed Bragg reflector (DBR)-tapered diode laser was already demonstrated. ${ }^{25}$ Such a diode-pumped Ti:sapphire laser has recently been demonstrated for optical coherence tomography and multiphoton microscopy. ${ }^{26,27}$ However, the output power was limited to below $200 \mathrm{~mW}$, which is less than that required for multimodal hyperspectral CARS imaging in backward direction propagation. Recently, a new concept for power scaling the SHG by cascading conversion stages was introduced. ${ }^{28}$ Using two nonlinear crystals, 39\% optical efficiency was achieved from a 10.5 W DBR-tapered diode laser. Such a laser could significantly boost the performance of diode-pumped femtosecond Ti:sapphire lasers to be used for multimodal hyperspectral CARS.

Here we present a multimodal nonlinear optical laser scanning microscope featuring simultaneous acquisition of hyperspectral CARS, SHG, and TPF in the backward propagation direction using a compact and potentially cost-effective femtosecond Ti:sapphire laser. The pump source combines a spectrally stabilized, high-efficient, and high-brightness tapered diode laser with periodically poled nonlinear crystals. The combination of the epi-detection scheme and the use of the abovementioned laser system allow to record $160 \times 160 \mu \mathrm{m}^{2}$ multimodal images of a fresh mouse ear tissue with a frame rate of more than $1 \mathrm{~Hz}$.

\section{Material and Methods}

\subsection{Compact Diode-Pumped Ti:Sapphire Laser}

As described by Hansen et al., ${ }^{28}$ the pump laser consists of a $6 \mathrm{~mm}$ DBR-tapered diode laser generating $10.5 \mathrm{~W}$ of light at $1064 \mathrm{~nm}$. Two separate electrical contacts control the currents through a 4-mm tapered diode section and half of a 2-mm ridge wave guide section. The DBR-tapered diode laser is mounted $p$-side up on a $\mathrm{CuW}$ heat spreader which is again mounted on a $25 \times 25 \mathrm{~mm}^{2}$ conduction-cooled package mount. The astigmatic emission from the diode is collimated by an aspheric lens in the fast axis and a cylindrical lens is added to correct the astigmatism and collimate the beam in the slow axis. The collimated beam is then sent through an optical isolator to prevent light from being reflected back to the DBR-tapered diode laser. A half-wave plate is used after the isolator to adjust the polarization and optimize the SHG. For efficient frequency doubling, a lens focuses the beam into a $40 \mathrm{~mm}$ periodically poled $\mathrm{MgO}$ doped lithium niobate (PPMgLN) crystal with a beam waist diameter of $\sim 80 \mu \mathrm{m}$. The crystal is antireflection coated at 1064 and $532 \mathrm{~nm}$ with temperature stabilization at $40^{\circ} \mathrm{C}$. The green and infrared beams are reimaged into a $40 \mathrm{~mm}$ long periodically poled MgO-doped stoichiometric lithium tantalate (PPMgSLT) crystal using two spherical mirrors and a glass plate compensates for phase shift between the two wavelengths. The SHG output power reaches up to $3.0 \mathrm{~W}$. The sealed housing of the DBR-tapered diode laser plus conversion stage (laser head) has a foot print of about $183 \times 114 \mathrm{~mm}^{2}$. The emission of this setup is used to pump a dispersive mirror based femtosecond laser. ${ }^{29}$ Our gain medium is a Ti:sapphire crystal with a 4-mm optical path. The pump beam having a collimated diameter of about $2.5 \mathrm{~mm}$ is focused into the crystal with a $35-\mathrm{mm}$ focal length AR-coated lens. Two dichroic curved mirrors are focusing and directing the beam to both arms of the $x$-folded cavity (Fig. 1). The angles are optimized to compensate for astigmatism inside the crystal. The intracavity dispersion is matched to support a sufficient bandwidth at optimized output power for simultaneous 


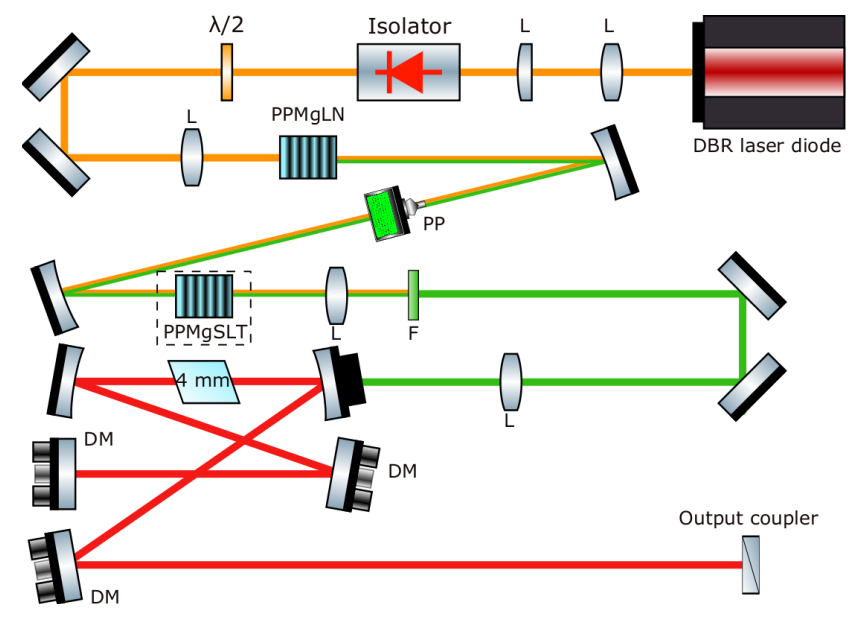

Fig. 1 Optical scheme of femtosecond laser light generation starting from a DBR-tapered diode laser. The black-dashed square denotes the additional conversion stage to enhance the second harmonic output. L, lenses; M, mirror; CM, curved mirror; PP, phase plate; F, filter; DM, dispersive mirror.

hyperspectral CARS, SHG, and TPF operation. In order to reduce the system size and complexity for improved reliability and stability, the repetition rate is increased compared to state-of-the-art femtosecond Ti:sapphire lasers applicable for CARS. Modelocked output power goes up to $540 \mathrm{~mW}$ with a full width at half maximum (FWHM) bandwidth of $\sim 15 \mathrm{~nm}$ centered at $805 \mathrm{~nm}$ with a repetition rate of $125 \mathrm{MHz}$ (Fig. 1). The Ti:sapphire laser and pump laser are temperature stabilized using a chiller.

\subsection{Epi-Detecting Multimodal Hyperspectral Microscope}

The above described laser system is used as a pump source for the epi-detecting multimodal hyperspectral setup shown in
Fig. 2. The multimodal nonlinear scanning microscope is capable to simultaneously detect backward-propagating TPF, SHG, and hyperspectral CARS signals generated within the focal volume. Point scanning is performed by a pair of galvanometric scanning mirrors $(6220 \mathrm{H} 8-\mathrm{mm}$ diameter, Cambridge Technology) and the sample position is controlled by a threedimensional motorized stage (PILine xy M687, PInano z P736, Physik Instrumente GmbH \& Co. KG). Moreover, CARS signals are generated by means of chirped pulses (the so-called hyperspectral or spectral focusing) to add a spectral dimension to the CARS image providing molecule-specific information of the specimen in question. This feature is of particular importance for biological and medical applications where the interpretation of the images, in most of the cases, is difficult due to the heterogeneous molecular composition of the sample.

The output beam of the compact diode-pumped Ti:sapphire femtosecond oscillator is split by a 60:40 beam splitter into the pump $(200 \mathrm{~mW})$ and the Stokes arm $(300 \mathrm{~mW})$. The light for the Stokes beam is then obtained by means of a polarization-maintaining photonic crystal fiber (PCF 5 from Klarskov et al..$^{30}$ ) which generates a broad continuum ranging from visible to nearinfrared spectral wavelengths. ${ }^{30}$ An $18 \mathrm{~nm}$ FWHM portion of a smooth broad peak generated around $1050 \mathrm{~nm}$ is filtered and subsequently amplified in a polarization maintaining singlemode ytterbium-doped fiber amplifier, to reach the desired pulse energy in the Stokes beam before recombining it with the pump beam. The amplified output for the Stokes beam has a maximum achievable output power of $130 \mathrm{~mW}$. To allow for full control of the amount of chirp, first both the pump and the Stokes pulses are compressed using dispersive mirrors to achieve the shortest pulse duration at the sample. While the pump pulses are compressed to $\sim 70 \mathrm{fs}$ at a center wavelength of $805 \mathrm{~nm}$, the Stokes pulses are compressed to $100 \mathrm{fs}$ at a center wavelength of $1050 \mathrm{~nm}$. Then, to introduce equal chirp on the pump and Stokes pulses in the simplest possible way, a

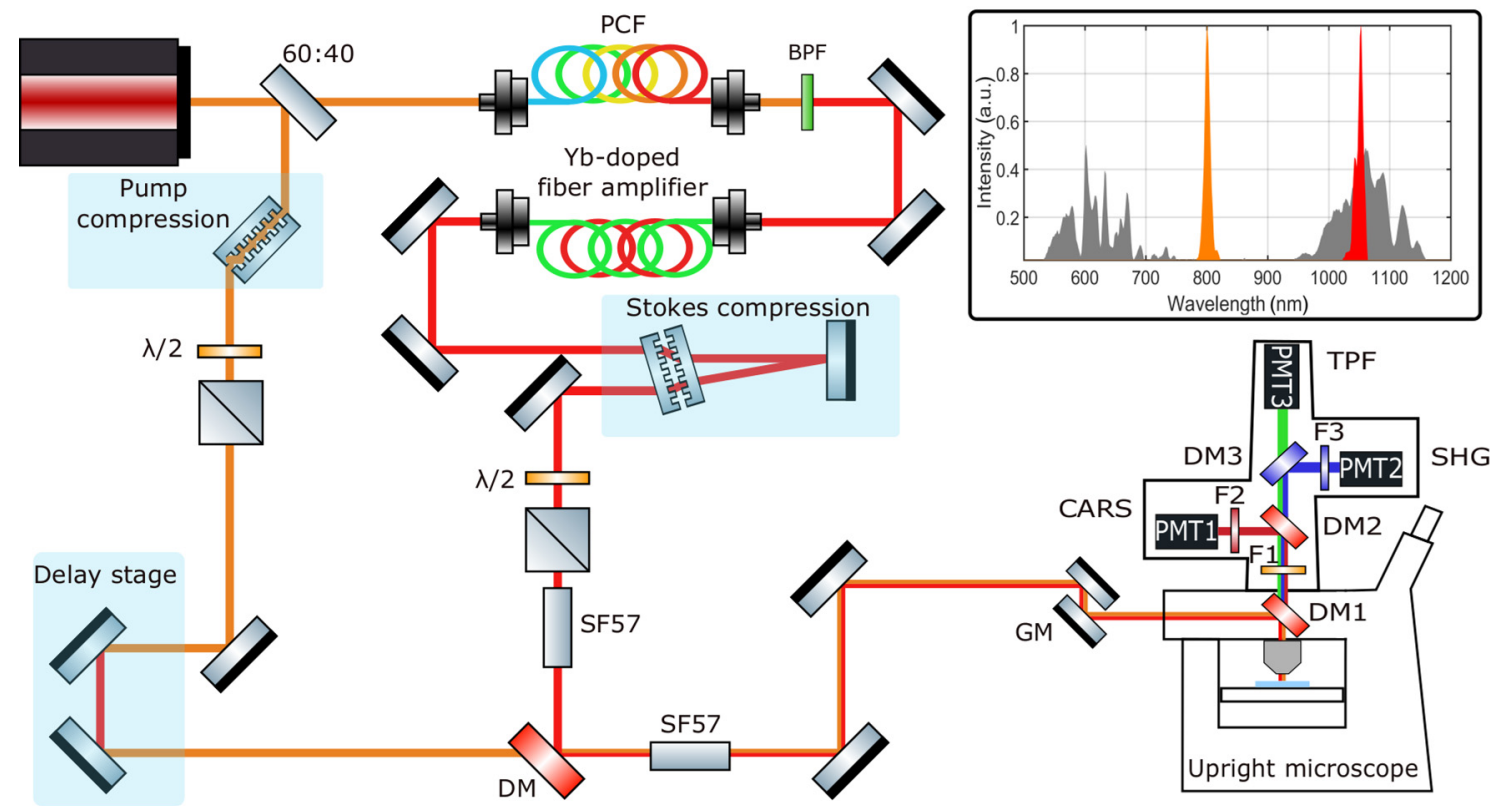

Fig. 2 Sketch of the compact multimodal imaging platform allowing epi-detection of CARS, SHG, and TPF signals. BPF, band-pass filter; PMT, photomultiplier tube; DM, dichroic mirror; GM, galvanometric scanning mirrors. Inset: The spectral data of the PCF output are shown in gray; pump (orange) and Stokes (red) spectra measured at the output of the compact diode-pumped Ti:sapphire oscillator and at the output of the $\mathrm{Yb}$-doped fiber amplifier, respectively. 
5-cm long block of SF57 glass is placed in the Stokes arm before being recombined, and after recombination both beams pass through a 10-cm long block of SF57 glass to achieve nearly matched chirps. The pump has no additional dispersive elements added before recombination. The chirped pulse durations of the pump and Stokes beams are around 1 and $0.7 \mathrm{ps}$, respectively. This difference in time duration is due to the different transformlimited bandwidths of the pump and the Stokes pulses. This configuration allows a spectral resolution for CARS spectroscopic microscopy of about $35 \mathrm{~cm}^{-1}$. The change in the temporal overlap is controlled by a computer-controlled delay stage allowing for fast tuning of the Raman frequency, covering the frequency range corresponding to the $\mathrm{C}-\mathrm{H}$ stretching vibrations. The pump and the Stokes pulses are focused on the sample using a water immersion objective (NIR Apo 40×, Nikon) with a numerical aperture of 0.8 . The epi-collection is performed by means of dichroic mirrors and photomultiplier tubes. The combination of a dichroic mirror (T680spxr, Chroma) and short-wave pass filter (RazorEdge 785, Semrock) decouples the excitation light from the generated signals and prevents leakage. Furthermore, two additional dichroic mirrors (T613spxxr, T412lpxt, Chroma) enable the simultaneous collection of CARS, TPF, and SHG. A band-pass filter (HQ640/50 m, Chroma) and a high-sensitive amplified photomultiplier tube (H7422-40, Hamamatsu) are used to collect the generated anti-Stokes signals. SHG and TPF are decoupled by means of a dichroic mirror (T412lpxt, Chroma). SHG is provided by an additional band-pass filter (BrightLine FF01-400/50-25, Semrock) and second amplified PMT (H10721-20, Hamamatsu). The last channel detects TPF with a similar detector head. Data acquisition is controlled by ScanImage 3.8 software. ${ }^{31}$

\section{Discussion and Results}

The performance of the presented compact multimodal nonlinear epi-detected microscope is demonstrated by recording hyperspectral images of a test sample consisting of a mixture of polystyrene and polymethyl methacrylate (PMMA) microspheres of 44- and $11-\mu \mathrm{m}$ diameter, respectively, immersed in water. The image shown in Fig. 3 is a projection of a 240 image dataset recorded along the spectral dimension from $\sim 2300$ to $\sim 3450 \mathrm{~cm}^{-1}$, corresponding to a time delay range between the pump and Stokes pulses of \pm 1.66 ps. Two different beads can be clearly identified in Fig. 3, wherein the contrast is obtained from the slightly different Raman resonances of the beads in the image, located at $2950 \mathrm{~cm}^{-1}$ for the PMMA and at $\sim 2930$ and $\sim 3050 \mathrm{~cm}^{-1}$ for the polystyrene beads. A spontaneous Raman spectrum of polystyrene and PMMA beads is shown for comparison. ${ }^{32}$ The FWHM of the spontaneous Raman signal from PMMA beads at the $\sim 2950 \mathrm{~cm}^{-1}$ band is $\sim 40 \mathrm{~cm}^{-1}$, allowing a crude estimate of our spectral resolution. With the degree of chirp set in our system, the measured FWHM of the $\sim 2950 \mathrm{~cm}^{-1}$ band is $\sim 70 \mathrm{~cm}^{-1}$, indicating a spectral resolution of $\sim 35 \mathrm{~cm}^{-1}$. Thus, our arrangement allows not only for spatial discrimination, but also spectral discrimination between PMMA and the polystyrene beads.

We further demonstrate CARS-based multimodal imaging of a biological tissue of interest for the study of sebaceous glands: a portion of mouse ear tissue was imaged ex vivo. Sebaceous glands are microscopic glands present in the skin of all mammalians that secrete a fatty substance, called sebum, onto the epithelial surface to lubricate and isolate the skin surface. The mode of secretion of the sebaceous glands is classified as holocrine since the sebum is released by the rupture of the plasma membrane-releasing holocrine, which is produced in the cytoplasm of the cells. The study of complex neuro-immune endocrine functions involved in the sebaceous glands has led to a growing interest in its physiology. ${ }^{33,34}$ There exist several related medical conditions in humans involving sebum, including acne vulgaris, sebaceous cyst, hyperplasia, and sebaceous adenoma which can be assessed by looking at the lipid content of the cells composing the gland using the multimodal nonlinear setup in Fig. 2. Therefore, the use of hyperspectral CARS to visualize

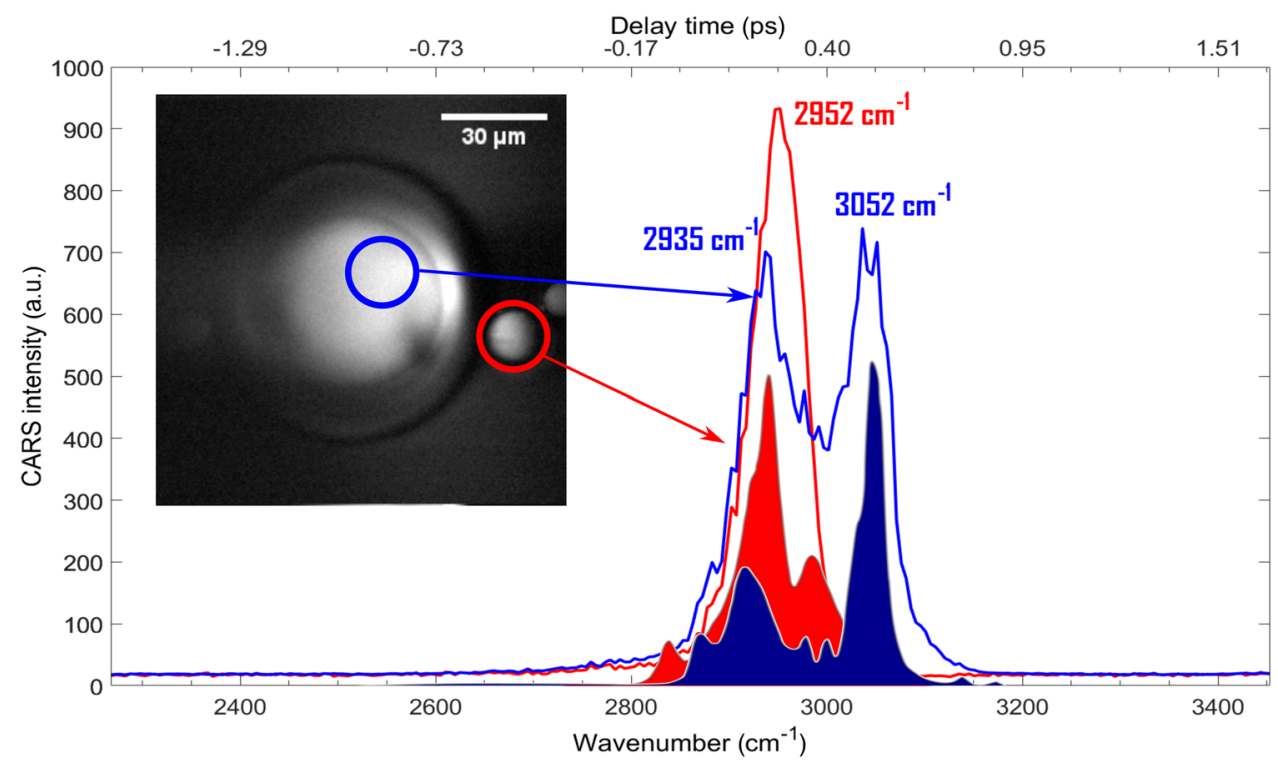

Fig. 3 Measured CARS spectrum of polystyrene (blue) and PMMA (red) beads obtained by scanning the time delay between the pump and Stokes pulses. For comparison, polystyrene and PMMA Raman spectra are shown in blue and red shaded area, respectively. ${ }^{32}$ Inset: Epi-detected CARS image of a mixture of polystyrene beads and PMMA suspended in water, $256 \times 256$ pixels, $6.4 \mu$ s pixel dwell time. The pump and Stokes powers at the sample were 19.2 and $6 \mathrm{~mW}$, respectively. 
the lipid content of these glands during their normal holocrine secretion process and in response to damage can give a labelfree way to study these types of diseases. ${ }^{34}$

A small portion of fresh mouse ear tissue was imaged within less than one hour after excision. We collected in the backward propagation direction the signals generated within the sample at different depths. The CARS signal in Fig. 4(b) shows sebocytes composing the sebaceous gland, which are clearly visible due to their high lipids content. Intracellular lipid lobules are visible as bright granules within each sebocyte, whereas nuclei and cell membranes are visible as dark structures due to their lower lipid content. The CARS signal from adipose tissue is shown in Fig. 4(c) where the fat cells are clearly identified. In Fig. 4(d) we show a CARS-based multimodal image of another sebaceous gland from the same tissue sample where epi-detected CARS, SHG, and TPF are represented with red, blue, and green colors, respectively. Also in this case, the epi-detected CARS signal shows sebaceous glands rich in lipids. The epi-detected SHG signal is generated from collagen composing the dermis layer of the skin tissue showing the collagen matrix supporting the sebaceous gland. TPF is generated through autofluorescence which arises mainly from elastin, a basic component of the extracellular matrix in the dermis layer. Despite the fact that backward-propagating signals in general are much weaker compared to forward-propagating signals, the energy of the femtosecond pulses delivered by the compact diode-pumped femtosecond laser system allows to obtain enough contrast from different structures in the mouse skin to clearly reveal the fat cells composing the adipose tissue, sebaceous glands, and collagen fibrils constituting the full collagen matrix. It is important to emphasize that all three signals are endogenous to the sample and no stains were used to enhance the contrast, i.e., label-free or stain-free imaging.

\section{Conclusion}

In conclusion, to the best of our knowledge, this is the first epidetected multimodal hyperspectral microscope platform using a compact diode-pumped Ti:sapphire femtosecond laser. This laser delivers more than $500 \mathrm{~mW}$ which is the highest output power ever reported from a compact non-solid-state laser pumped

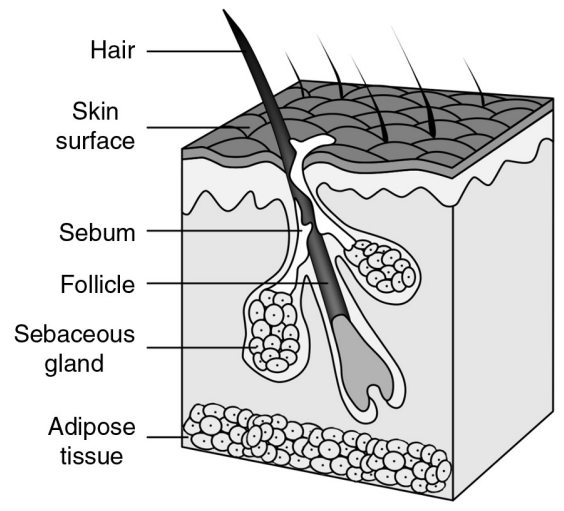

(a)

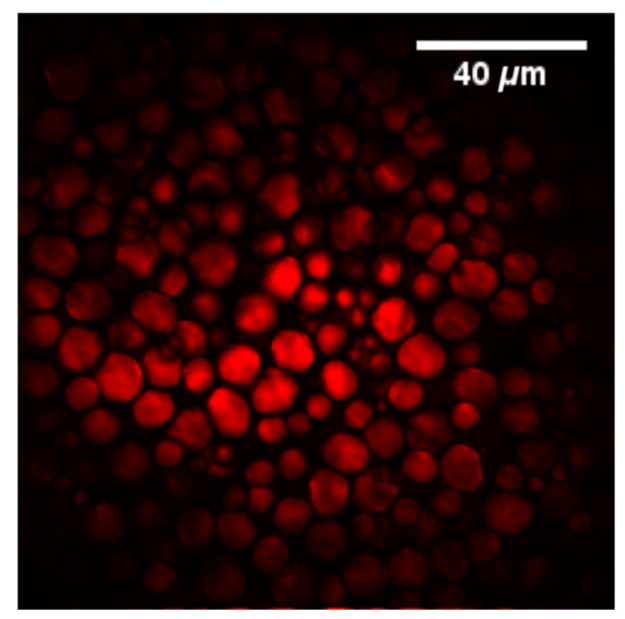

(c)

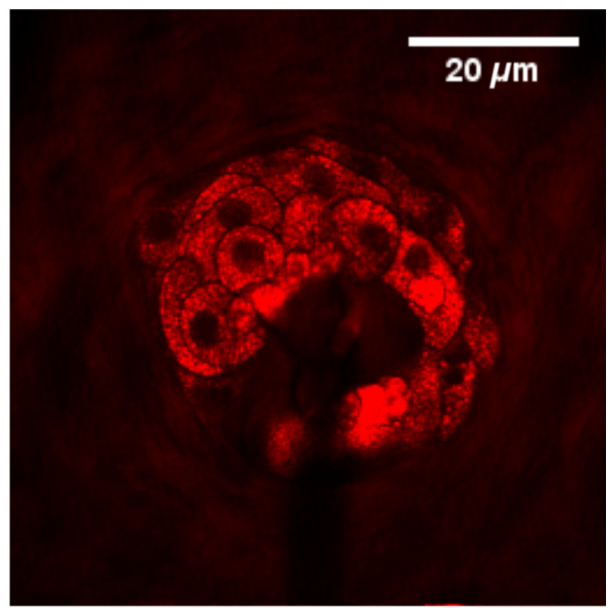

(b)

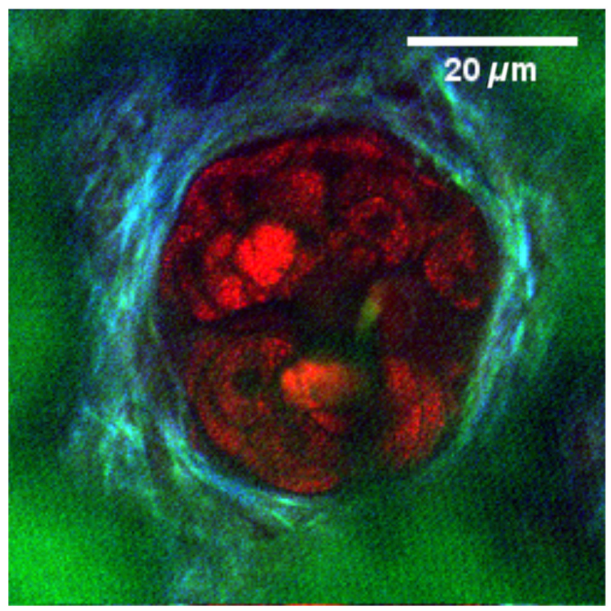

(d)

Fig. 4 (a) A simplified sketch showing the composition of skin tissue. (b), (c) and (d) Multimodal images of a mouse ear tissue ex vivo at $2845 \mathrm{~cm}^{-1}$ using the compact multimodal platform. (b) Epi-detected CARS image of a sebaceous gland (30- $\mu \mathrm{m}$ deep into the tissue). (c) Epi-detected CARS image of adipose tissue (60- $\mu \mathrm{m}$ deep into the tissue). (d) Epi-detected CARS (red), SHG (blue), and TPF (green) signals (30- $\mu \mathrm{m}$ deep into the tissue). All images are $256 \times 256$ pixels, $6.4 \mu$ s pixel dwell time. The pump and Stokes powers at the sample were 19.2 and $6 \mathrm{~mW}$, respectively. 
femtosecond Ti:sapphire laser. Since this light source makes use of a frequency-doubled DBR-tapered diode pump laser, it not only ensure compactness, cost-effectiveness, and reduced power consumption compared to conventional Ti:sapphire lasers, but can also be designed to emit at a specific wavelength range between 700 and $900 \mathrm{~nm}$. The imaging capability of the system was tested by imaging SHG, TPF, and CARS signals in the C-H stretching vibrational region from a mixture of polystyrene and PMMA beads and from fresh unstained mouse ear tissue. By means of spectral focusing, pump and Stokes pulses were equally chirped, providing a spectral resolution for CARS spectroscopic microscopy of $\sim 35 \mathrm{~cm}^{-1}$. The images presented here demonstrate the potential of our approach for a compact, practical, and high performance CARS-based multimodal microscope (CARS, SHG, and TPF). The obtained image quality is comparable to the image quality achieved with previous demonstrations, suggesting that this imaging platform could pave the way for use in routine clinical environment.

\section{Disclosures}

No conflicts of interest, financial or otherwise, are declared by the authors.

\section{Acknowledgments}

This work was supported by the European Union's Seventh Framework Programme (FAMOS, FP7-ICT-2011-3-5a) under grant agreement $n$ FP7-ICT-317744. The authors acknowledge the contribution of Danny Noordegraaf from Norlase ApS for technical assistance and Lasse Leick from NKT Photonics for providing photonic crystal fiber.

\section{References}

1. W. Denk, J. Strickler, and W. Webb, "Two-photon laser scanning fluorescence microscopy," Science 248, 73-76 (1990).

2. A. Zumbusch, G. R. Holtom, and X. S. Xie, "Three-dimensional vibrational imaging by coherent anti-stokes Raman scattering," Phys. Rev. Lett. 82, 4142-4145 (1999).

3. P. J. Campagnola et al., "High-resolution nonlinear optical imaging of live cells by second harmonic generation," Biophys. J. 77, 3341-3349 (1999).

4. W. R. Zipfel, R. M. Williams, and W. W. Webb, "Nonlinear magic: multiphoton microscopy in the biosciences," Nat. Biotechnol. 21, 13691377 (2003).

5. T. T. Le et al., "Label-free molecular imaging of atherosclerotic lesions using multimodal nonlinear optical microscopy," J. Biomed. Opt. 12(5), 054007 (2007).

6. J. P. Pezacki et al., "Chemical contrast for imaging living systems: molecular vibrations drive CARS microscopy," Nat. Chem. Biol. 7, 137-145 (2011).

7. K. König et al., "Optical skin biopsies by clinical CARS and multiphoton fluorescence/SHG tomography," Laser Phys. Lett. 8, 465-468 (2011).

8. H. G. Breunig et al., "Clinical coherent anti-Stokes Raman scattering and multiphoton tomography of human skin with a femtosecond laser and photonic crystal fiber," Laser Phys. Lett. 10, 025604 (2013).

9. M. Weinigel et al., "Multipurpose nonlinear optical imaging system for in vivo and ex vivo multimodal histology," J. Med. Imaging 2, 016003 (2015).

10. J.-X. Cheng et al., "An epi-detected coherent anti-Stokes Raman scattering (E-CARS) microscope with high spectral resolution and high sensitivity," J. Phys. Chem. B 105, 1277-1280 (2001).

11. A. Volkmer, J.-X. Cheng, and X. S. Xie, "Vibrational imaging with high sensitivity via epidetected coherent anti-Stokes Raman scattering microscopy," Phys. Rev. Lett. 87, 023901 (2001).

12. C. L. Evans et al., "Chemical imaging of tissue in vivo with video-rate coherent anti-Stokes Raman scattering microscopy," Proc. Natl. Acad. Sci. U.S.A. 102, 16807-16812 (2005).
13. J.-X. Cheng, "Coherent anti-Stokes Raman scattering microscopy," Appl. Spectrosc. 61, 197-208 (2007).

14. S. Yue, M. Slipchenko, and J.-X. Cheng, "Multimodal nonlinear optical microscopy," Laser Photonics Rev. 5, 496-512 (2011).

15. A. F. Pegoraro et al., "Optimally chirped multimodal CARS microscopy based on a single Ti:sapphire oscillator," Opt. Express 17, 2984 (2009).

16. R. Selm et al., "Ultrabroadband background-free coherent anti-Stokes Raman scattering microscopy based on a compact Er:fiber laser system," Opt. Lett. 35, 3282 (2010).

17. Y. J. Lee et al., "Optimized continuum from a photonic crystal fiber for broadband time-resolved coherent anti-Stokes Raman scattering," Opt. Express 18, 4371 (2010).

18. T. Hellerer, A. M. Enejder, and A. Zumbusch, "Spectral focusing: high spectral resolution spectroscopy with broad-bandwidth laser pulses," Appl. Phys. Lett. 85(1), 25-27 (2004).

19. I. Rocha-Mendoza, W. Langbein, and P. Borri, "Coherent anti-Stokes Raman microspectroscopy using spectral focusing with glass dispersion," Appl. Phys. Lett. 93(20), 201103 (2008).

20. T. T. Le, S. Yue, and J.-X. Cheng, "Shedding new light on lipid biology with coherent anti-Stokes Raman scattering microscopy," J. Lipid Res. 51, 3091-3102 (2010).

21. M. Baumgartl et al., "All-fiber laser source for CARS microscopy based on fiber optical parametric frequency conversion," Opt. Express 20, 4484 (2012).

22. B. Nie et al., "Multimodal microscopy with sub-30 fs Yb fiber laser oscillator," Biomed. Opt. Express 3, 1750 (2012).

23. E. S. Lamb and F. W. Wise, "Multimodal fiber source for nonlinear microscopy based on a dissipative soliton laser," Biomed. Opt. Express 6, 3248 (2015).

24. O. B. Jensen et al., " $15 \mathrm{~W}$ green light generation by single-pass second harmonic generation of a single-frequency tapered diode laser," Opt. Express 17, 6532 (2009).

25. A. Müller et al., "Frequency-doubled DBR-tapered diode laser for direct pumping of Ti:sapphire lasers generating sub-20 fs pulses," Opt. Express 19, 12156 (2011).

26. A. Unterhuber et al., "Simultaneous dual wavelength eye-tracked ultrahigh resolution retinal and choroidal optical coherence tomography," Opt. Lett. 38, 4312 (2013).

27. K. König et al., "Multiphoton imaging with a novel compact diodepumped Ti:sapphire oscillator," Microsc. Res. Tech. 78, 1154-1158 (2015).

28. A. K. Hansen et al., "Concept for power scaling second harmonic generation using a cascade of nonlinear crystals," Opt. Express 23, 15921 (2015).

29. L. Xu et al., "High-power sub-10-fs Ti:sapphire oscillators," Appl. Phys. B Lasers Opt. 65, 151-159 (1997).

30. P. Klarskov et al., "Supercontinuum generation for coherent anti-Stokes Raman scattering microscopy with photonic crystal fibers," Opt. Express 19, 26672 (2011).

31. T. A. Pologruto, B. L. Sabatini, and K. Svoboda, "ScanImage: flexible software for operating laser scanning microscopes," Biomed. Eng. Online 2, 13 (2003).

32. Sigma-Aldrich, "Raman spectrum of polystyrene and PMMA beads," 2016, http://www.sigmaaldrich.com/.

33. R. Nejati, C. Skobowiat, and A. T. Slominski, "Commentary on the practical guide for the study of sebaceous glands," Exp. Dermatol. 22, 629-630 (2013).

34. Y. Jung et al., "Longitudinal, 3D in vivo imaging of sebaceous glands by coherent anti-Stokes Raman scattering microscopy: normal function and response to cryotherapy," J. Invest. Dermatol. 135, 39-44 (2015).

Marco Andreana received his $\mathrm{PhD}$ from Université de Limoges and the Università degli studi di Brescia in 2011. In 2015, he joined the Center for Medical Physics and Biomedical Engineering at the Medical University of Vienna, Austria, as a postdoc scholar, where he is currently working on nonlinear microscopic systems for biophotonics. His current research interests include nonlinear frequency conversion, nonlinear microscopy, and multimodal imaging for biomedical applications.

Tuan Le is a senior staff scientist at Femtolasers Produktions $\mathrm{GmbH}$. $\mathrm{He}$ received his $\mathrm{MSc}$ degree in physics and his $\mathrm{PhD}$ in mechanical engineering from the Vienna University of Technology. Since joining 
Femtolasers in 1998, his research interests have been femtosecond lasers and their applications.

Anders K. Hansen received his MSc and $\mathrm{PhD}$ degrees in physics in 2010 and 2013, respectively, working on laser cooling of molecular ions in the Ion Trap Group at Aarhus University. In 2013, he joined DTU Fotonik at the Technical University of Denmark as a postdoc scholar, where he is currently a researcher working on laser systems for biophotonics. He has a special interest in nonlinear frequency conversion, laser diodes, and phase manipulation of light.

Aart $\mathbf{J}$. Verhoef received his MSc degree in physics and astronomy from the Vrije Universiteit Amsterdam, the Netherlands, in 2002, and his $\mathrm{PhD}$ in experimental physics from the Ludwig-MaximiliansUniversität München, Germany, in 2007. Currently, he is working as an assistant professor at the Center for Medical Physics and Biomedical Engineering at the Medical University of Vienna, Austria. His current research interests include the development of femtosecond all-fiber oscillators and amplifiers, nonlinear optics, as well as the development of advanced methods for multiphoton microscopy and neuroimaging.

Ole B. Jensen received his MSc degree in applied physics from the Technical University of Denmark (DTU), Lyngby, Denmark, in 1999 and his PhD in optics from DTU in 2002. Currently, he is a senior researcher at the Department of Photonics Engineering, DTU Fotonik. His current research interests include diode lasers, solidstate lasers, and nonlinear frequency conversion as well as biomedical applications of laser systems.

Peter E. Andersen received his MSc and PhD degrees from the Technical University of Denmark, Denmark, 1991 and 1994, respectively. His current research interests include laser systems, optical coherence tomography, nonlinear microscopy, and multimodal imaging for biomedical applications.
Paul Slezak received his medical doctor degree at the Medical University of Vienna in 2009. He is currently a senior researcher at the Ludwig Boltzmann Institute for Experimental and Clinical Traumatology, where he heads the imaging department and manages the soft tissue regeneration research group. He has extensive experience in various animal models and has a further research focus on extracorporeal shock wave therapy and local hemostasis.

Wolfgang Drexler was a full professor of biomedical imaging at Cardiff University, UK, from 2006-2009. Since 2009, he has been a full professor and the head of the Center for Medical Physics and Biomedical Engineering at the Medical University of Vienna, Austria. He spent 2 years at MIT, Cambridge, USA, received the Austrian START Award (2001) and the COGAN Award (2007), published more than 180 publications, gave more than 220 invited/keynote talks, and accomplished 11 million research grant income.

Alma Fernández studied physics at Universidad Nacional de Panamá and Engineering Physics at the Carl von Ossietzky Universität Oldenburg and University of Applied Sciences in Emden, Germany. She received her PhD in experimental physics from the Ludwig-Maximilians-Universität München, Germany, in 2007. Currently, she is a senior researcher at the Photonics Institute of the TU Wien, Austria. Her current research interests include the development of novel femtosecond all-fiber oscillators and amplifiers, novel nonlinear frequency conversion schemes, and biomedical applications of femtosecond lasers.

Angelika Unterhuber has 20 years of experience in ultrafast optics. She received her $\mathrm{PhD}$ from Vienna University of Technology and worked as an R\&D engineer in different laser-related companies. From 2006 to 2009, she was at School of Optometry and Vision Sciences at Cardiff University. Currently, she works at the Medical University of Vienna, focusing on development of novel light sources and their biomedical application in the field of OCT and nonlinear imaging. 